\title{
EVALUATION OF THE RELATIONSHIP BETWEEN PULMONARY ARTERY STIFFNESS AND RIGHT VENTRICULAR FUNCTION IN PATIENTS WITH BEHÇET'S DISEASE USING TRANSTHORACIC ECHOCARDIOGRAPHY
}

Salim Yaşar ${ }^{1}$, Murat Unlu ${ }^{2}$, suat gormel ${ }^{1}$, Emre Yalcinkaya ${ }^{3}$, Baris BUGAN ${ }^{1}$, Yalçın Gökoğlan ${ }^{1}$, Erkan Yıldırım ${ }^{1}$, Serdar Fırtına ${ }^{1}$, Serkan Asil ${ }^{1}$, Murat CELIK ${ }^{1}$, Uygar Yuksel ${ }^{1}$, Hasan Kabul ${ }^{1}$, Muhammet Çınar ${ }^{1}$, and cem barcin ${ }^{1}$

${ }^{1}$ Ankara Gulhane Egitim ve Arastirma Hastanesi

${ }^{2}$ Department of Cardiology Private Tekden Hospital Denizli/Turkey

${ }^{3}$ Department of Cardiology Sancaktepe Şehit ProfDr Illhan Varank Training and Research Hospital İstanbul/Turkey

November 6, 2021

\begin{abstract}
Background: Behçet's disease is a systemic vasculitis that can affect all sizes of arteries and veins. Arterial stiffness is a term used to describe the visco-elastic properties of vessel wall. In this study, we aimed to evaluate the relationship between pulmonary artery stiffness (PAS) and the right ventricular (RV) functions in asymptomatic Behçet's patients with no cardiovascular risk factors. Methods: We studied 40 patients who were diagnosed by the international diagnostic criteria of Behçet's disease and 40 healthy individuals who were matching demographic properties with the patients. Two groups were matched by age, gender, clinical history and other clinical features. Substantial medical history concerning the factors that can affect right ventricle diastolic function (such as medications, smoking status, other comorbities, etc.) was taken and general physical examination was carried out. The right and left ventricular functions as well as valvular functions were evaluated by using echocardiography. Also Two-dimensional, M-mode, pulsed wave (PW) Doppler echocardiographic parameters were measured for right ventricular functions. PW Doppler flow trace was obtained from the pulmonary valve with regards to pulmonary artery stiffness. Results: There was no significant difference in terms of clinical and demographic properties. No statistically significant difference was found upon comparison of the left ventricular end of systole and diastole diameters, the diastolic and systolic thicknesses of the interventricular septum (IVS) and left ventricular posterior wall (LVPW), left atrium (LA) diameter and left ventricular ejection fraction $(\mathrm{LVEF})$ values $(\mathrm{p}>0.05)$ of the two groups. Right ventricular myocardial performance index (MPI) value was found higher in Behçet's patients and a statistically significant difference was detected between the groups $(\mathrm{p}<0.01)$. Tricuspid annular plane systolic excursion (TAPSE) values were found to be statistically significantly lower in the patient group as compared to the control group $(\mathrm{p}<0.01)$. In PW Doppler measurements, early passive filling $(\mathrm{E})$ wave flow velocity and $\mathrm{E} / \mathrm{A}$ ratio were found to be statistically significantly lower, deceleration time (DT) was higher in the patient group ( $<<0.01$ ). In Behçet's patients without clinical pulmonary involvement, the pulmonary artery systolic pressure (PASP) was found to be statistically significantly higher in the patient group $(\mathrm{p}<0.01)$. The values of pulmonary artery stiffness (PAS) were found to be significantly higher in the patient group $(\mathrm{p}<0.01)$. The relationship between the right ventricular function markers and PAS were evaluated in the patient group. There was no statistically significant relationship between PAS and MPI and TAPSE. But there was a significant correlation between PAS and PASP and duration of illness $(\mathrm{p}<0.001$ and $\mathrm{r}=0.682 ; \mathrm{p}=0.047$ and
\end{abstract}


$\mathrm{r}=0,316)$. Conclusion: Behçet's patients without cardiac symptoms and signs, reduction in right ventricular functions and increase in PAS was detected. Although there is no correlation between right ventricular functions and PAS, increased PAS may be an early marker of reduction of the right ventricular functions. Consequently, routine cardiological examination and detailed evaluation of biventricular functions by using echocardiography should be greatly beneficial in Behcet's patients, even though there are no signs or symptoms.

\section{INTRODUCTION}

Behçet's disease (BD) is a chronic relapsing systemic inflammatory disease of unknown etiology. The disease, which is characterized by recurrent orogenital ulceration and uveitis, was first described in 1937 by a dermatologist, Prof. Dr. Hulusi Behçet; in 1947, the disease was acknowledged in the medical literature by the name Behçet's syndrome, Morbus Behçet, or BD []. Cardiovascular involvement is a common condition in which the cardiac structure and vascular elements are concurrently affected, and it can be seen in $7 \%-45 \%$ of patients. Vascular involvement is among the major complications observed during the course of BD. The disease can affect the arteries and veins of any size. However, the venous system is more frequently affected than the arterial system []. In the literature, $85 \%$ venous involvement has been reported and found to be more common in males $(40 \%)$ than in females $(5 \%)[3,4,5,6]$. The lower extremity veins are most commonly involved [], and the entire vein is affected, with no segmental involvement [7, 8].

In studies evaluating cardiac functions of patients with BD in whom substantial cardiac involvement is not seen, mostly data regarding the left ventricle were obtained, and data regarding right ventricular (RV) functions are limited. Although this may be attributed to various reasons, the most important one is that the evaluation of RV functions is quite challenging due to the complex structure and asymmetric shape of the right ventricle [9]. Although there are limited studies in the literature, it has been reported that RV functions may be affected in BD. Moreover, the role of pulmonary artery stiffness (PAS) in the deterioration of RV functions is not fully elucidated. Arterial stiffness is a term used to describe the viscoelastic properties of the vessel wall. It refers to the loss of elastic tissue in the vessel wall and may be associated with increased afterload []. It has been reported that increased stiffness in the central veins increases the energy requirement of the right ventricle for each contraction, affects the arterial mechanics (pulsatile driving pressure) and flow dynamics, consequently, negatively affects the ventricular performance [11].

In this study, we aimed to evaluate the relationship between PAS and RV functions using transthoracic echocardiography in patients with asymptomatic BD and without substantial cardiac involvement.

\section{MATERIALS AND METHODS}

\section{Patient Group}

The study included 40 (2 female, 38 male) healthy volunteers and 40 patients ( 2 female, 38 male) who were diagnosed with BD according to the diagnostic criteria of the International Study Group between October 2014 and April 2015 and are still under follow-up and treatment and show no cardiac involvement. The patients were informed about the study and their consent was obtained.

Before performing echocardiographic evaluation, the patients' detailed medical history was evaluated, and all the relevant systems were examined. Both the groups were compared based on clinical characteristics and medical history. The control group individuals did not have a history of known disease or disease requiring drug use, whereas the patients with BD did not have a history of any other known systemic disease except their current disease.

Active disease was defined as having at least one finding during the evaluation according to the diagnostic criteria. Patients who were in the active phase and had cardiac symptoms and signs were not included in the study. None of the patients with normal electrocardiography findings were using drugs (beta blockers, angiotensin-converting-enzyme inhibitors/angiotensin receptor blockers, calcium channel blockers, etc.) that could affect cardiac functions during echocardiographic evaluation.

\section{Echocardiographic Evaluation}


The patients included in the study were examined using a Philips IE33 6.0 (Philips Medical Systems, Andover, MA, USA) echocardiography device. All the patients' pulse rates and blood pressure values measured before echocardiography were within the normal limits. After resting for $10 \mathrm{~min}$, the patients were placed in the left lateral decubitus position, and echocardiographic findings were recorded during superficial respiration. Measurements were performed by a single physician. During the recordings, the patients' ECG was monitored. Three consecutive measurements were obtained for each parameter using a 2.5-MHz probe, and the average values were recorded. Patients whose images had a poor quality and those with inaccessible data were excluded from the study.

First, the pericardium, valve morphologies, and wall movements were evaluated using the M-mode and twodimensional echocardiography. The M-mode imaging was performed in accordance with the recommendations of the American Society of Echocardiography. Left ventricular end-diastolic and end-systolic diameters, fractional shortening, ejection fraction using M-Mode imaging, the interventricular septum's systolic and diastolic thicknesses, left ventricular posterior wall's systolic and diastolic thicknesses, and left atrial diameter were measured.

Pulsed wave (PW) Doppler echocardiography was performed in accordance with the recommendations of the American Society of Echocardiography. Using the apical four-chamber view and placing the sample volume at the tip of the tricuspid valve leaflets, early (E) and late (A) diastolic flow velocities and deceleration times were measured, and $\mathrm{E} / \mathrm{A}$ ratios were calculated.

Tricuspid annular plane systolic excursion (TAPSE) was measured using the M-mode imaging findings of the RV annular segment's systolic motion through the lateral free wall of the tricuspid annulus in the apical four-chamber view (Figure 1).

Intervals required for RV myocardial performance index (MPI), also known as Tei index, were calculated using PW Doppler. The MPI value was calculated by dividing the sum of the cardiac isovolumic relaxation and contraction times by the ejection time (Figure 2).

Right atrial pressure was considered to be $3 \mathrm{mmHg}$, and using the Bernoulli equation, pulmonary artery systolic pressure (sPAP) was calculated for evaluating the tricuspid flow-mediated Doppler data obtained from the patients.

$\mathrm{sPAP}=4 \mathrm{v}^{2}+$ right atrial pressure (v: peak tricuspid regurgitation rate)

Pulmonary artery acceleration time was calculated using the trace of PW Doppler sample volume positioned in the pulmonary artery $1 \mathrm{~cm}$ distal to the pulmonary valve annulus in the parasternal short-axis view. PAS was calculated as the ratio of maximum systolic shift frequency obtained from Doppler tracing to acceleration time $(\mathrm{HZ})(\mathrm{kHz} / \mathrm{s})$ (Figure 3).

\section{Statistical Evaluation}

The data obtained from the patients were transferred into a computer. Data analysis was conducted using the SPSS (Statistical Package for Social Sciences) 22.0 package program. Number, percentage, mean, standard deviation, median, and minimum and maximum values were used for presenting descriptive variables. The conformity of continuous variables to a normal distribution was evaluated using Kolmogorov-Smirnov test. For comparisons between groups, t-test was used for continuous variables with a normal distribution, and Mann-Whitney U test was used for variables without a normal distribution. Chi-square test was used to compare discrete variables. Pearson's correlation coefficient was used to evaluate the linear relationship between variables. A $p$ value of $<0.05$ was considered significant in all tests.

\section{RESULTS}

\section{Demographic characteristics}

A total of 40 patients with BD who met the inclusion criteria were included in the study. Forty healthy gender- and age-matched individuals were included as controls in the study. As can be seen in Table 1, no 
significant difference was found between the two groups in terms of age, gender, body mass index, systolic and diastolic blood pressures, and resting heart rates (Table 1).

\section{Clinical Findings}

It was determined that the mean disease duration of the patients with BD was $7.72 \pm 5.65$ years (minimum 1 year, maximum 24 years). The mean age of disease onset was $25.77 \pm 6.46$ (minimum 12 years, maximum 36 years). In addition to the history of oral aphthous lesion, $24(60 \%)$ patients had a history of genital aphthous lesion, $29(72.5 \%)$ had a history of papulopustular lesion, and $6(15 \%)$ had a history of erythema nodosumlike lesion. In addition to mucocutaneous involvement, there was ocular involvement in $13(32.5 \%)$ patients, joint involvement in $23(57.5 \%)$ patients, neurological involvement in $1(2.5 \%)$ patient, and gastrointestinal system involvement in $3(7.5 \%)$ patients. None of the patients had vascular involvement. Pathergy test was positive for $27(67.5 \%)$ of the 40 patients, and human leukocyte antigen-B51 was positive for $25(62.5 \%)$ patients (Table 2).

\section{Echocardiography Findings}

Both the groups were compared in terms of two-dimensional and M-mode imaging findings for the left cardiac structures. No significant difference was found in the interventricular septum's end-systolic and end-diastolic thicknesses, LV end-systolic and end-diastolic diameters, left atrial dimensions, and ejection fraction and fractional shortening values, which are systolic function parameters $(\mathrm{p}>0.05)$ (Table 3).

No significant difference was found between the two groups in terms of right atrial and RV diameters, RV endsystolic and end-diastolic diameters, and RV fractional area change measured from the apical four-chamber view $(\mathrm{p}>0.05)$ (Table 4$)$.

Furthermore, the groups were compared in terms of PW Doppler parameters of the right ventricle. The mean diastolic early peak flow velocity (E) value of the tricuspid valve was significantly greater in the control group $(59.43 \pm 4.45)$ compared to the patient group $(57.83 \pm 5.07)(\mathrm{p}<0.001)$. The comparison of late diastolic peak flow velocities (A) showed that the mean value of the patient group $(43.74 \pm 3.65)$ was higher than that of the control group $(39.09 \pm 5.23)$, and the difference was significant $(\mathrm{p}<0.001)$ (Table 4$)$.

The $\mathrm{E} / \mathrm{A}$ ratio of the tricuspid valve was found to be lower in the patient group than in the control group. A significant difference was found between the two groups in terms of E/A ratio $(p<0.001)$ (Table 4).

In terms of deceleration time measured by PW Doppler, the patient group had significantly higher values (216.85 \pm 25.09$)$ compared to the control group (197.40 \pm 14.91$),(\mathrm{p}<0.001)$ (Table 4).

Although RV ejection time in the patient group was found to be higher compared to that in the control group, no significant difference was observed. The sum of RV isovolumetric contraction and relaxation times was significantly higher in the patient group $(92.75 \pm 8.68)$ compared to the control group $(73.67 \pm 9.44)$, and a significant difference was observed $(\mathrm{p}<0.001)$ (Table 4$)$.

MPI values, which were used in the assessment of the global cardiac RV systolic and diastolic functions, measured using PW Doppler were significantly higher in the patient group $(0.30 \pm 0.02)$ than in the control group $(0.24 \pm 0.03)$, and there was a significant difference $(\mathrm{p}<0.001)$ (Table 4) (Figure 4).

TAPSE values were $23.85 \pm 1.42$ in the patient group and $27.78 \pm 1.12$ in the control group. A significant difference was found between the two groups $(\mathrm{p}<0.001)$ (Table 4) (Figure 5).

When the sPAP values were compared between the two groups, the mean SPAP value of the patients with BD $(27.80 \pm 3.13)$ was found to be significantly higher than that of the control group individuals $(20.25$ $\pm 2.89)(\mathrm{p}<0.001)$ (Table 4). PAS was found to be higher in the patient group $(8.80 \pm 1.11)$ than in the control group $(7.09 \pm 0.71)$, and this differences was significant $(\mathrm{p}<0.001)$ (Table 4) (Figure 6).

On evaluating the correlation between PAS and RV function markers in the patient group, sPAP was found to be correlated with PAS (Figure 7). In the correlation analysis, disease duration was also correlated with PAS (Figure 8) (Table 6). 
In addition, the relationship of MPI and TAPSE, two of the most frequently used values in the evaluation of global RV functions, with PAS was evaluated in the patient group. As shown in Table 5, an increase in PAS was associated with an increase in MPI and a decrease in TAPSE in the patient group; however, there was no statistical significance $(\mathrm{p}=0.703$ and $\mathrm{p}=0.912$, respectively) (Table 7$)$.

\section{DISCUSSION}

In our study, the relationship between PAS and RV functions, which may be an indicator of subclinical cardiovascular involvement, was evaluated by transthoracic echocardiography in patients with asymptomatic $\mathrm{BD}$ without cardiovascular risk factors. In the literature, pericarditis, myocarditis, endocarditis with valvular insufficiency, intracardiac thrombus, endomyocardial fibrosis, coronary arteritis presenting with or without myocardial infarction, and coronary artery and sinus of valsalva aneurysms have been reported related to cardiac involvement in patients with BD. In a study of 807 patients, cardiac involvement was detected in $6 \%$, pericarditis in $38 \%$, endocarditis often accompanied by aortic valve insufficiency in $27 \%$, intracardiac thrombus in $20 \%$, myocardial infarction in $17 \%$, endomyocardial fibrosis in $8 \%$, and myocardial aneurysm in $2 \%$ of the patients [12].

Historically, our knowledge of RV function in healthy individuals and in those with diseases has been limited compared to that of LV function. Quantitative evaluations of RV in pathological events affecting the cardiovascular system are neglected because RV has less muscle mass, is limited in its function to pump blood only to the pulmonary system, and less frequently affected by diseases such as myocardial ischemia, cardiomyopathy, or valvulopathy. Therefore, less attention has been paid on detecting or measuring RV dysfunction. Similar to other diseases, this is also the case in the echocardiographic evaluation of RV in patients with BD. Thus, our study aimed to perform a separate and detailed echocardiographic evaluation of the right ventricle in patients with $\mathrm{BD}$.

PAS is a novel echocardiographic parameter used for noninvasive assessment of the elastic properties of the pulmonary vascular bed [13]. Although it is known that aortic stiffness has an important role in predicting cardiovascular mortality and morbidity, it is believed that PAS may also indicate the status of the pulmonary vascular bed. In some studies, a correlation was found between PAS and pulmonary artery pressure and elasticity. However, most of the studies have been conducted using invasive methods. Invasive techniques complicate the use of this new parameter in clinical studies. In our study, PAS formulated based on the calculation of aortic stiffness was assessed via echocardiography. Although it has been previously reported that decreased pulmonary artery compliance may cause deterioration in RV myocardial functions [14], our study is the first to evaluate the relationship between RV functions and PAS in patients with BD.

PAS can be calculated by dividing the maximal systolic shift frequency by the acceleration time obtained from pulmonary artery Doppler flow tracing. The validity of this noninvasive method in determining PAS was previously demonstrated by Görgülü et al. $[15,16]$.

Duman et al.[17] investigated the relationship between PAS and RV functions in patients with cardiovascular asymptomatic systemic lupus erythematosus and showed increased RV MPI, which reflects global RV function, as well as increased PAS and sPAP in the group of patients with systemic lupus erythematosus. In addition, they suggested that PAS was significantly associated with decreased RV functions and independent of variables such as age and disease activity index.

In our study, the relationship between PAS and RV functions in patients with BD was evaluated using transthoracic echocardiography. It was observed that PAS was significantly increased in patients with BD compared to healthy controls. Although MPI was found to be significantly higher and TAPSE was lower in the patient group than in the control group, no significant correlation was found in the correlation analyses evaluating the relationship of these parameters with PAS. The lack of statistical significance between them may be due to the small number of patients included in our study. On the other hand, correlation analysis between SPAP and PAS and disease duration and PAS demonstrated that SPAP was strongly correlated with PAS $(\mathrm{r}=0.682 ; \mathrm{p}<0.001)$, whereas disease duration was moderately correlated $(\mathrm{r}=0.316 ; \mathrm{p}=0.047)$. 
Studies support that PAS is the major determinant of ventricular loading and the compliance of the pulmonary artery is a significant factor in the transmission of RV load to the left heart [18]. It has been reported that the increase in stiffness in the central veins increases the energy required for each contraction of the right ventricle since it affects arterial mechanics (pulsatile driving pressure) and flow dynamics and, consequently, worsens the ventricular performance.[11]. In light of this information and based on the results of this and other studies mentioned above, it was concluded that PAS increase may be a strong predictor of the development of RV dysfunction.

\section{LIMITATIONS OF THE STUDY}

The most important limitation of our study is the small number of patients. The small sample size is mainly due to the exclusion of all patients with a history or diagnosis of diseases that may cause impaired RV function, making it difficult to enroll more patients. Another limitation of the study is the lack of use of right heart catheterization, which is the gold standard for evaluating RV functions, and the use of Doppler echocardiography, which is a noninvasive method, instead. However, as shown in previous studies, Doppler parameters used to evaluate cardiac functions show good correlation with invasive test parameters. Thus, considering the potential risks and cost of right heart catheterization as an invasive procedure, echocardiography, a non-invasive method, was preferred for use in patients with asymptomatic cardiovascular disease.

\section{CONCLUSION AND RECOMMENDATIONS}

In conclusion, it was determined that asymptomatic BD caused a decrease in RV functions. The obtained data suggested that BD affected global RV functions by causing subclinical myocardial involvement on the background of endothelial dysfunction. Our study is valuable since it shows subclinical cardiac involvement in patients with asymptomatic BD through comparison with healthy individuals and supports the results of the literature.

Another important finding is that pulmonary hypertension is more common in patients with BD than in healthy individuals. Since there is no other underlying cause that can explain pulmonary hypertension, it has been proposed that patients with BD without remarkable symptoms may have subclinical pulmonary involvement. To better elucidate these findings, it would be more beneficial to evaluate these parameters using a larger sample size and with two different groups including patients with $\mathrm{BD}$ in the exacerbation and remission periods.

Considering the effects of dysfunction on the clinical course and outcomes of the disease, it has been suggested that the assessment of RV functions, sPAP, and PAS are of great importance in the evaluation of prognosis in patients with BD. Accordingly, it is thought that routine cardiology examination and further evaluation of the aorta, valvular pathologies, and left as well as RV functions by conventional and tissue Doppler echocardiography examinations will be considerably helpful in patients with $\mathrm{BD}$ even if there are no active complaints.

In line with the findings of our study, it was considered that increased PAS that is likely to be caused by subclinical vascular involvement in the pulmonary artery of patients with BD may lead to the development of subclinical RV dysfunction. Nevertheless, prospective-controlled studies with larger numbers of patients and long-term clinical follow-up are needed to reveal the relationship between PAS and RV function and the impact of this relationship on prognosis.

\section{CONFLICT OF INTEREST}

The authors declare that they have no conflict of interest.

Figure 1: Calculation of TAPSE using the M-mode imaging through the lateral tricuspid annulus in the apical four-chamber view.

Figure 2: Calculation of right ventricular myocardial performance index (RV MPI) using PW Doppler. The "a" interval refers to the time (in ms) between the closure and opening of the tricuspid valve; the "b" interval 
indicates systolic ventricular ejection time (ET) of the right ventricle. RV MPI is calculated by dividing the difference between the "a" and "b" intervals by the "b" interval [17].

Figure 3: Calculation of pulmonary artery stiffness (PAS). (PAS = MSS/AT; MSS: Maximum Standard Shift Frequency; AT: Acceleration Time)

Figure 4. Comparison of the patient and control groups in terms of myocardial performance index values

Figure 5. Comparison of TAPSE values in the patient and control groups

Figure 6. Comparison of pulmonary artery stiffness values in the patient and control groups

Figure 7. The relationship between pulmonary artery systolic pressure and pulmonary artery stiffness (p $<0.001, \mathrm{r}=0.682$ )

Figure 8. Relationship between disease duration and pulmonary artery stiffness $(\mathrm{p}=0.047, \mathrm{r}=0.316)$

\section{REFERENCES}

1 .Dilsen, N., History and development of Behcet's disease. Rev Rhum Engl Ed, 1996. 63 (7-8): p. 512-9.

2. James, D.G. and A. Thomson, Recognition of the diverse cardiovascular manifestation in Behcet's disease. Am Heart J, 1982. 103 (3): p. 457-8.

3. Tunc, R., et al., Cerebral venous thrombosis is associated with major vessel disease in Behcet's syndrome. Ann Rheum Dis, 2004. 63 (12): p. 1693-4.

4. Koc, Y., et al., Vascular involvement in Behcet's disease. J Rheumatol, 1992. 19 (3): p. 402-10.

5. Sarica-Kucukoglu, R., et al., Vascular involvement in Behcet's disease: a retrospective analysis of 2319 cases. Int J Dermatol, 2006. 45 (8): p. 919-21.

6. Melikoglu M, U.S., Tascilar K et al Large vessel involvement in Behçet's syndrome: a retrospective survey. An Rheum Dis, 2008. (Suppl II):67.

7. Fukuda, Y., et al., Pathological studies on Behcet's disease. Ryumachi, 1980. 20 (4): p. 268-75.

8. Matsumoto, T., T. Uekusa, and Y. Fukuda, Vasculo-Behcet's disease: a pathologic study of eight cases. Hum Pathol, 1991. 22 (1): p. 45-51.

9. Nagel, E., M. Stuber, and O.M. Hess, Importance of the right ventricle in valvular heart disease. Eur Heart J, 1996. 17 (6): p. 829-36.

10. Vural MG, S.H., Evaluation of pulmonary artery stiffness by transthoracic echocardiography, and its role in the early diagnosis of pulmonary artery aneurysm in patients with Behçet's disease. Archives of the Turkish Society of Cardiology, 2011.

11. JK., L., The arterial circulation: physical principles and clinical applications.. Totowa, NJ: Humana Press, 2000 .

12. Geri G, Wechsler B, Thi Huong D Le, Isnard R, Piette J-C, Amoura Z, et al. Spectrum of cardiac lesions in Behçet disease: a series of 52 patients and review of the literature. Medicine. 2012 Jan;91 (1):25-34.

13 Pasiersky TJ, S.R., Binkley PF,Pearson AC., Echocardiografic evaluation of pulmonary artery distensibility. Chest, 1993. 1080 (3): p. 103.

14. Fourie PR, Coetzee AR, Bolliger CT. Pulmonary artery compliance: its role in right ventricular-arterial coupling. Cardiovasc Res 1992;26;839-44

15. Görgülü S, Eren M, Yildirim A, Ozer O, Uslu N, Celik S, Dağdeviren B, Nurkalem Z, Bağirtan B, Tezel T. A new echocardiographic approach in assessing pulmonary vascular bed in patients with congenital heart disease: pulmonary artery stiffness. The Anatolian Journal of Cardiology. 2003 Jun;3 (2):92-7. 
16.Gorgulu S, Eren M, Uslu N, Ozer O, Nurkalem Z. The determinants of right ventricular function in patients with atrial septal defect. Int J Cardiol. 2006 Jul 28;111 (1):127-30.

17. Dr.Dursun Duman,D.S.M.,Dr. Refik Demirtunç Dr. Bilgehan Karadağ. Increased pulmonary artery stiffness and its relation to right ventricular function in patients with systemic lupus erythematosus. Archives of the Turkish Society of Cardiology, 2008. 36: p.82-89

18. Kass DA. Ventricular arterial stiffening; integratingthe pathophysiology. Hypertension 2005;46:185-93
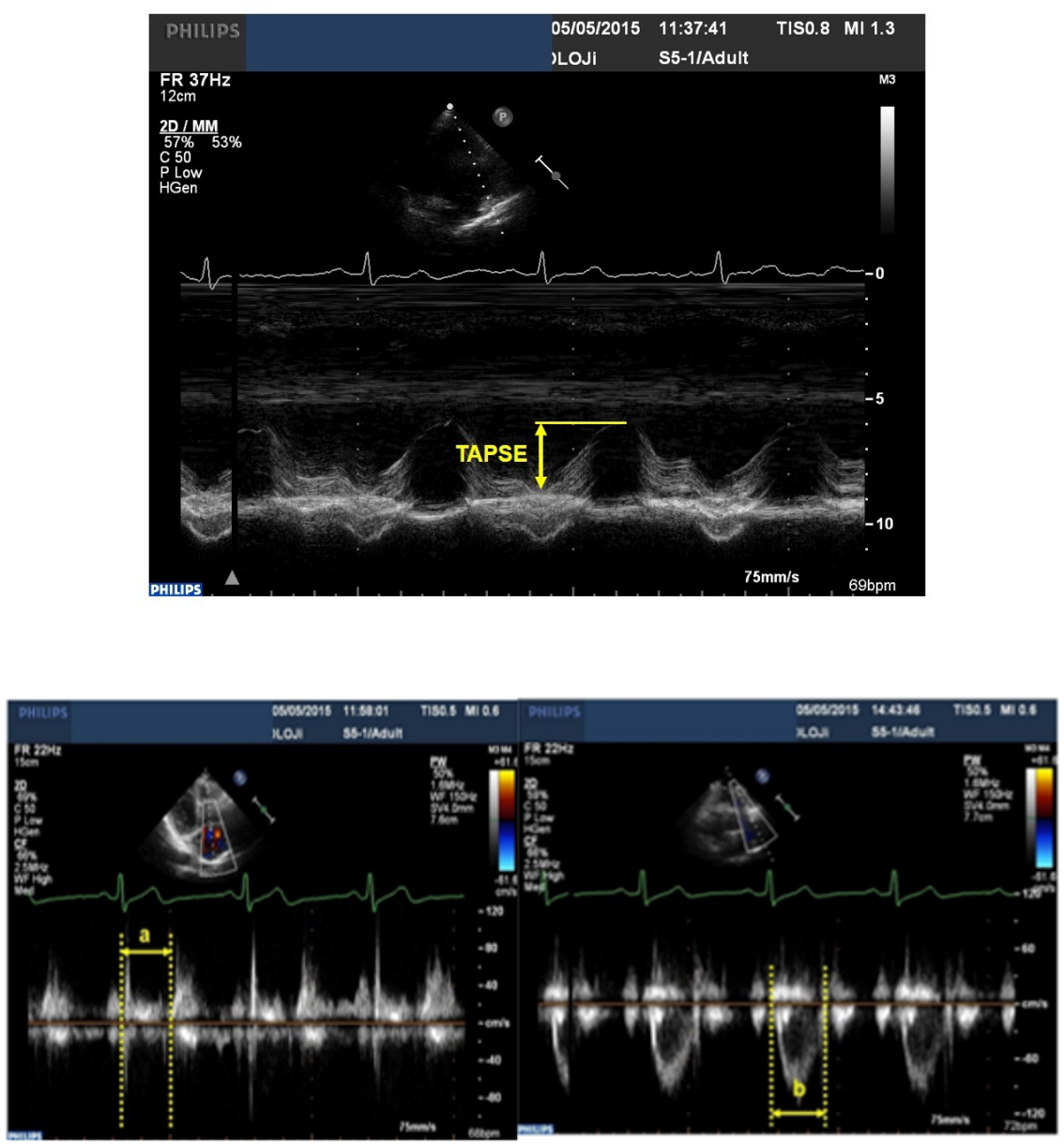

RV MPi $=a-b / b$ 

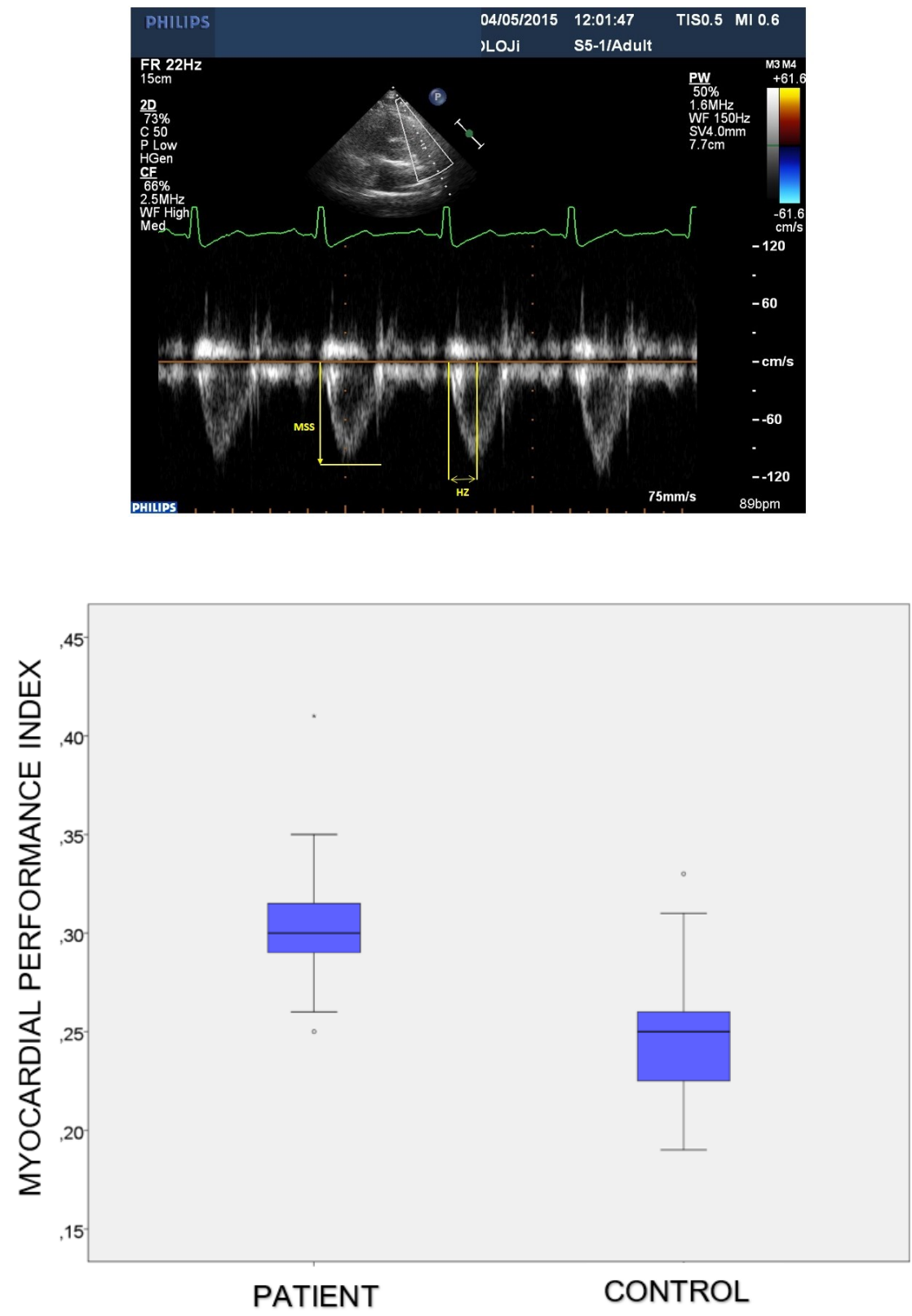

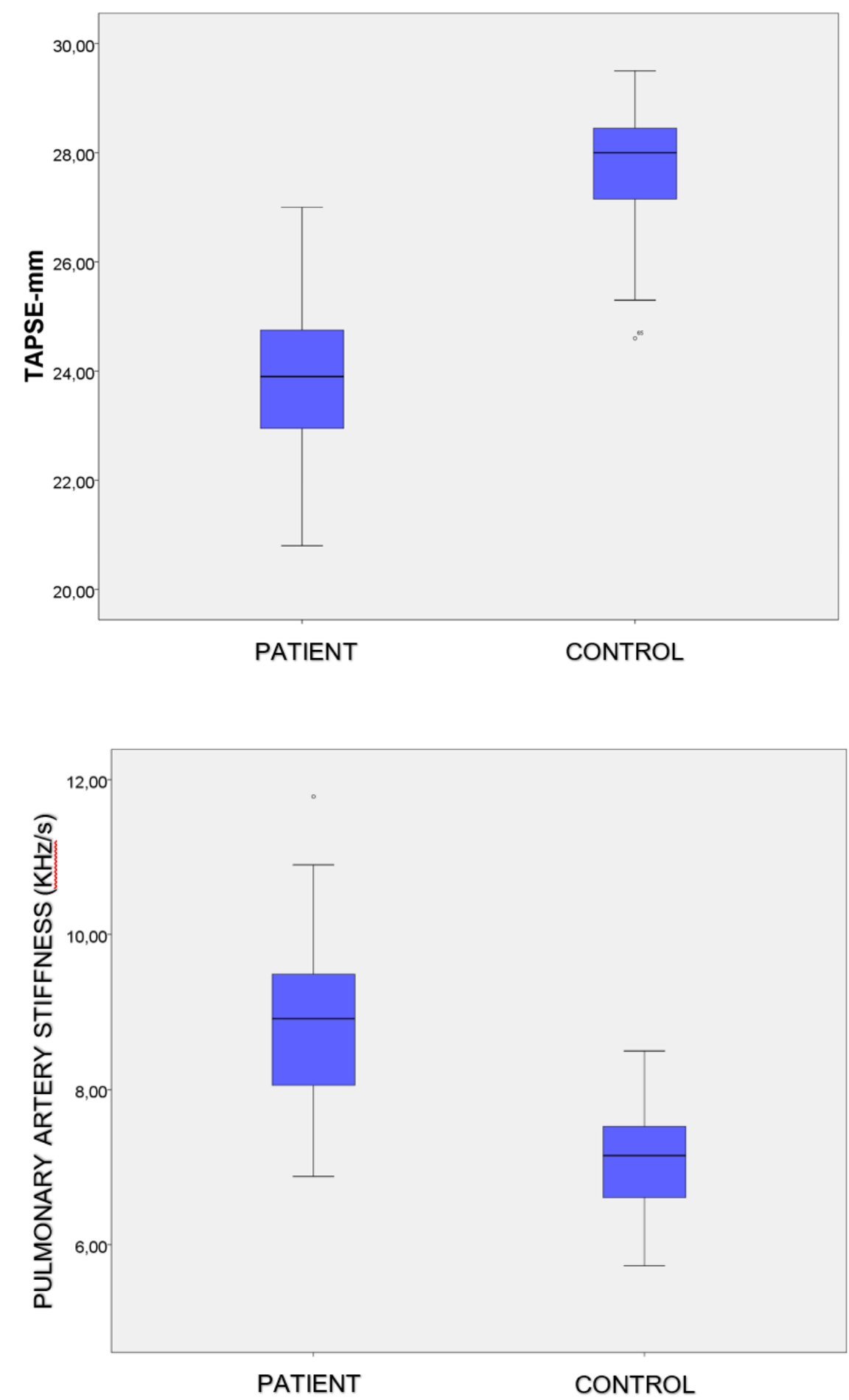

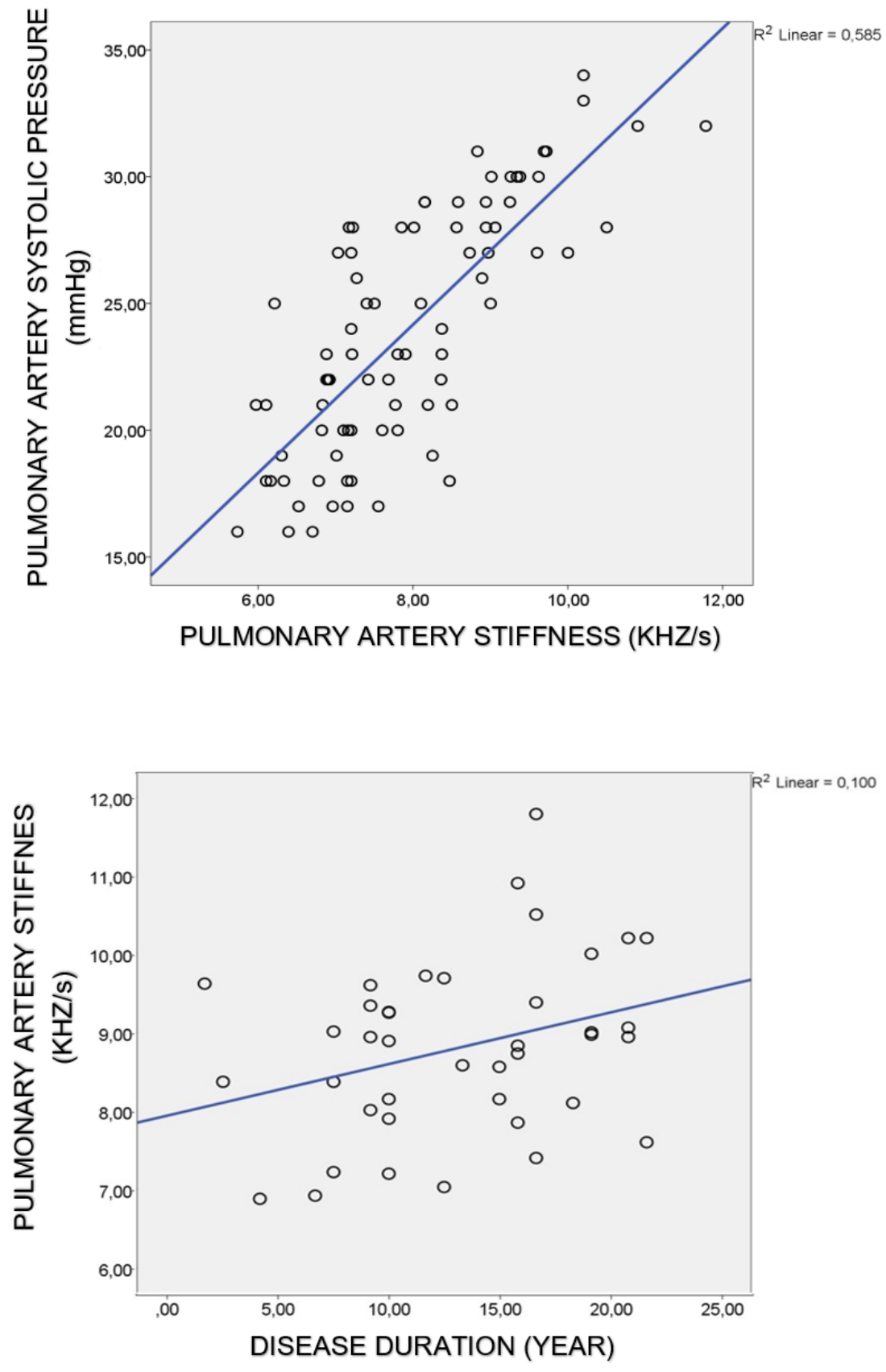

\section{Hosted file}

Table 1.docx available at https://authorea.com/users/444707/articles/544402-evaluation-ofthe-relationship-between-pulmonary-artery-stiffness-and-right-ventricular-function-inpatients-with-beh\%C3\%A7et-s-disease-using-transthoracic-echocardiography

\section{Hosted file}

Table 2.docx available at https://authorea.com/users/444707/articles/544402-evaluation-of- 
the-relationship-between-pulmonary-artery-stiffness-and-right-ventricular-function-inpatients-with-beh\%C3\%A7et-s-disease-using-transthoracic-echocardiography

\section{Hosted file}

Table 3.docx available at https://authorea.com/users/444707/articles/544402-evaluation-ofthe-relationship-between-pulmonary-artery-stiffness-and-right-ventricular-function-inpatients-with-beh\%C3\%A7et-s-disease-using-transthoracic-echocardiography

\section{Hosted file}

Table 4.docx available at https://authorea.com/users/444707/articles/544402-evaluation-ofthe-relationship-between-pulmonary-artery-stiffness-and-right-ventricular-function-inpatients-with-beh\%C3\%A7et-s-disease-using-transthoracic-echocardiography

\section{Hosted file}

Table 5.docx available at https://authorea.com/users/444707/articles/544402-evaluation-ofthe-relationship-between-pulmonary-artery-stiffness-and-right-ventricular-function-inpatients-with-beh\%C3\%A7et-s-disease-using-transthoracic-echocardiography

\section{Hosted file}

Table 6.docx available at https://authorea.com/users/444707/articles/544402-evaluation-ofthe-relationship-between-pulmonary-artery-stiffness-and-right-ventricular-function-inpatients-with-beh\%C3\%A7et-s-disease-using-transthoracic-echocardiography

\section{Hosted file}

Table 7.docx available at https://authorea.com/users/444707/articles/544402-evaluation-ofthe-relationship-between-pulmonary-artery-stiffness-and-right-ventricular-function-inpatients-with-beh\%C3\%A7et-s-disease-using-transthoracic-echocardiography 